\title{
Validación de rúbrica para evaluación de e-actividades diseñadas para el logro de competencias digitales docentes
}

\section{Validation of rubric for evaluation of e-activities designed for achievement of digital teaching competences}

\author{
Beatriz Marcano $^{11}$, Victoria Íñigo ${ }^{2}$, José Manuel Sánchez Ramírez ${ }^{3}$ \\ Universidad Internacional De La Rioja, España ${ }^{123}$ \\ (iD) Orcid ID: https://orcid.org/0000-0003-2461-75771 \\ (iD) Orcid ID: https $/ /$ orcid.org/0000-0001-6037-6307² \\ (iD) Orcid ID: https://orcid.org/0000-0002-5891-1283
}

Recepción: 15 de agosto de 2019

Aceptación: 09 de febrero de 2020

\begin{abstract}
Resumen
Debido a la importancia de desarrollar competencias digitales docentes en los procesos de formación del profesorado, se diseñaron e-actividades a fin de incrementar la funcionalidad de dichas competencias y ser evaluadas mediante rúbricas. Por ello, el objetivo de este estudio es verificar la validez de las rúbricas a fin de valorar la competencia digital docente y su respectiva efectividad, según el modelo del Instituto Nacional de Tecnologías Educativas y Formación del Profesorado de España. A tal efecto, se implementó un procedimiento de validación en dos fases: una por juicio de expertos, en la que debían corroborar la correspondencia de las rúbricas con los indicadores de la competencia digital docente y otra fase de validación empírica para validar la eficiencia de las rúbricas para las correcciones de e-actividades, corregidas por tres profesores. En esta fase se realizó un análisis de diferencia de medias (F de Friedman al nivel de 0.05 ) y se encontró que no había diferencias significativas entre los correctores que no fueron el profesor de la asignatura. Además, se concluyó que las rúbricas eran válidas para comprobar las competencias digitales docentes evaluadas.
\end{abstract}

Palabras clave: Competencia digital docente, profesorado, validación por juicio de expertos, rúbricas, e-actividades, tecnologías de la información y la comunicación.

${ }^{1}$ Correspondencia al autor:

e-mail: beatriz.marcano@unir.net 


\begin{abstract}
Due to the importance of developing digital teaching competencies in the teacher training processes, e-activities were designed in order to increase the functionality of these competences and to be evaluated through rubrics. Therefore, the objective of this study is to verify the validity of the rubrics in order to assess the digital teaching competence and their respective effectiveness, according to the model of the National Institute of Educational Technologies and Teacher Training in Spain. To this end, a two-phase validation procedure was implemented: one by expert judgment, in which the correspondence of the rubrics with the indicators of digital teaching competence had to be corroborated and another phase of empirical validation to validate the efficiency of the rubrics for e-activity corrections, corrected by three teachers. In this phase, a mean difference analysis was performed (Friedman's Test at the 0.05 level) and it was found that there were no significant differences between the correctors who were not the teacher of the subject. Furthermore, it was concluded that the rubrics were valid to check the evaluated digital teaching competences.
\end{abstract}

Keywords: Digital competence for teachers, teaching, validation by expert judgment, rubrics, e-activities, information and communication technologies.

\title{
Introducción
}

El impulso de las competencias digitales docentes en un máster de formación del profesorado es un reto ineludible al que se debe responder con eficacia. Sus estudiantesfuturos profesores a su vez-, deberán dar respuesta a las exigencias que se les presenten en las aulas con unos alumnos cada vez más inmersos en los formatos de comunicación digital (Sánchez-Otero et al, 2019; Ferrando, Hurtado y Beltrán, 2018). Por tanto, desarrollar habilidades y destrezas para el diseño de las actividades de aula acorde a esos formatos es una tarea que aún está pendiente de parte del profesorado (Area, 2015; Area y González,2015; Álvarez y Gisbert 2015; Pérez-Escoda, y Rodríguez-Conde, 2016; Tourón y Martin, 2019). Atentos a esta necesidad en el máster de formación del profesorado de secundaria, bachillerato y formación profesional, se hace pertinente incluir en sus actividades formativas una serie de exigencias que apunten al desarrollo de competencias digitales docentes. Se entiende por estas al conjunto de habilidades, destrezas y maneras de responder ante las exigencias pedagógicas y didácticas, con el apoyo de herramientas tecnológicas de información y de comunicación. Desde esta perspectiva, se diseñaron una serie de actividades formativas para el fomento del aprendizaje y mejora de las competencias digitales docentes.

En los programas de formación competencial en entornos virtuales es muy propicio dar relevancia al diseño de las e-actividades (actividades en entorno online) por constituirse en piedra angular en torno a las cuales se desarrollarán las competencias deseables, y más si se trata de formar competencias digitales. En este sentido, las e-actividades deben poseer ciertas 
capacidades: ser funcionales, estar adaptadas al futuro entorno laboral, favorecer la colaboración, y apuntar a la solución de problemas como estrategia transferible a otros entornos (Silva, 2017; Blau, Grinberg y Shamir-Inbal, 2018). De esta manera, se favorece el "saber hacer en contexto" y la cualificación laboral y profesional (Silva y Mazuera, 2019).

En cuanto al diseño de las e-actividades, se tomó como punto de referencia la propuesta del Instituto Nacional de Tecnologías Educativas y de Formación del Profesorado (INTEF) de España), el cual establece cinco áreas competenciales: Información y alfabetización informacional, Seguridad, Comunicación y colaboración, Resolución de problemas y Creación de contenido digital (INTEF, 2017). Para cada una de estas, se elaboraron una serie de indicadores del nivel de desarrollo de cada área de la competencia digital. La evaluación del nivel de desarrollo de la competencia digital docente (CDD), ha inspirado diversos instrume ntos de evaluación, muchos de ellos apoyados en la propuesta de las áreas del INTEF y sus indicadores. (Martín et al, 2016; Durán, Gutiérrez, y Paz, 2016; Salazar-Gómez, Tobón y Juárez-Hernández, 2018, Touron et al, 2018).

Se estableció que la valoración de las actividades formativas planteadas a los estudiantes del máster fuera a través de rúbricas de evaluación. La rúbrica se ha reconocido como un instrumento de evaluación idóneo para una diversidad de tareas, incluidas las cotidianas; y su pertinencia en la evaluación de competencias, permite disgregar las tareas complejas que componen una competencia en microtareas más observables (Pérez y Flores, 2017). Con estas, se guía la ruta a seguir por el estudiante en cada criterio de evaluación, más si se comparten desde el inicio del curso (Alsina et al, 2013; Brown, 2015). Los criterios de evaluación deben estar alineados con las competencias a desarrollar, lo que imprime validez y solidez al proceso formativo (García-Ross, 2011). Este es el patrón definido en el establecimiento de la validez de las rúbricas de evaluación de las actividades formativas con las que se pretende fomentar el desarrollo de la CDD.

En este orden de ideas, se apela a la opinión de Escobar-Pérez y Cuervo-Martínez (2008), quienes destacan la importancia de usar métodos de validez de contenidos de ítems que puedan garantizar la rigurosidad en las investigaciones sociales a través de los procedimientos estadísticos pertinentes. Para la validación de contenidos en educación, el método del juicio de expertos resulta muy oportuno, pues permite la contrastación de acuerdos entre especialistas del área y aporta un criterio objetivo para calibrar instrumentos de evaluación. 
En este sentido, se planteó conocer en qué medida las rúbricas que se utilizaban para evaluar las actividades corresponden con las diferentes áreas de formación en la competencia digital docente, y en qué medida son efectivas como rúbricas de evaluación. Para dar respuesta a esta doble interrogante se diseñó un proceso de doble validación que se explica en el apartado de metodología y que responde a los objetivos de esta investigación. Como objetivo general se bosquejó el análisis y validación de la correspondencia de las rúbricas de evaluación de las eactividades de la asignatura "Complementos de la especialidad de Tecnología e informática" del máster de Formación del Profesorado con las áreas e indicadores de la competencia digital docente según el modelo INTEF 2017 y su efectividad para la corrección de las e-actividades. En cuanto a los objetivos específicos, estos se dividen en dos fases: en primer lugar, se busca determinar la validez de las rúbricas de evaluación de las e-actividades y su correspondencia para avalar el desarrollo de las competencias digitales docentes según el modelo del INTEF 2017; en segundo lugar, se intenta validar la efectividad de rúbricas con las que se evalúan las e-actividades de formación en competencia digital docente.

\section{Métodos y materiales}

\section{Las actividades formativas}

Se seleccionaron las cinco actividades propuestas para la evaluación continua de la asignatura de "Complementos de Formación de la especialidad de Tecnología e Informática" en la maestría de Formación del profesorado, en una universidad online de España. Las actividades consistían en:

- Actividad 1: Búsqueda, selección y evaluación de una experiencia didáctica relacionada con el fomento de vocaciones STEM (Ciencia, Tecnología, Ingeniería y Matemáticas según sus siglas en inglés). En esta actividad se pide a los estudiantes que indaguen sobre algunas iniciativas que, a nivel educativo y formativo en general, se están desarrollando en el entorno de educación secundaria y relacionadas con: robótica educativa, programación, creación de aplicaciones móviles (apps), creación de videojuegos, etc. Luego de aplicar un análisis FODA, se elabora un documento con dicho análisis y las conclusiones del estudiante. Este documento se sube a alguna plataforma de publicación online de acceso gratuito y su enlace se comparte mediante un tuit en la red social Twitter y un hashtag específico para esa tarea. 
- Actividad 2: Con esta actividad se pretende que el estudiante analice la funcionalidad de comunicación para el entorno educativo que ofrece Twitter en su doble rol: microblogging y red social. Además, se busca la autorreflexión del estudiante sobre las ventajas que tiene para su propia autoformación. La actividad conlleva por parte del estudiante idear una alternativa de uso de Twitter para sus futuros alumnos en la especialidad de Tecnología e Informática que incluya el uso de un hashtag particular para la actividad. Como en la anterior actividad, el estudiante de maestría lo pone por escrito y lo sube a cualquier plataforma de publicación online de libre acceso; luego publica el enlace en Twitter con el hashtag \#Msec_TeI_Twitter, y tuitea el enlace por el "envío de actividades" para su respectiva evaluación.

- Actividad 3: Construir un entorno personal de aprendizaje (PLE) propio de cada estudiante, al cual deben añadir recursos y organizarlos según los siguientes aspectos:

a) Buscar y organizar información: herramientas y estrategias de lectura y fuentes de información; colaboración en su clasificación y almacenamiento.

b) Publicar y crear contenidos: herramientas TIC y estrategias de revaloración de la información: los entornos o servicios en los que puede transformar la información (sitios donde escribe, comenta, analiza, recrea, pública).

c) Comunicar: herramientas y estrategias de relación, entornos donde se relaciona con otras personas de/con las que aprende.

El alumno elige alguna de las herramientas digitales presentadas en el curso (por ejemplo, Symbaloo) y edita su PLE, el cual debe presentarlo añadiendo una exposición de las ventajas, dificultades, obstáculos, etc. que ha encontrado en el momento de diseñar su PLE, así como una breve conclusión de cuáles son las utilidades y aplicaciones que tiene su uso en las aulas de secundaria.

- Actividad 4: Diseño y creación colaborativa de una webquest para implementar el aprendizaje basado en proyectos en estudiantes de secundaria. Esta es la actividad más compleja, ya que requiere que los estudiantes se organicen en grupos de trabajo, acuerden la temática que abordarán y establezcan las normas de colaboración y trabajo grupal, además de determinar los medios y canales de comunicación virtual que utilizarán. Para la edición de la webquest también se utilizarán herramientas de edición y plataformas en la nube. 
- Actividad 5: Se plantea diseñar el desarrollo de un tema implementando la metodología flipped classroom, según la cual el docente prepara el contenido para que el alumno lo estudie antes de ir a clase apoyados en recursos y herramientas tecnológicas. Posteriormente, en el aula se desarrollan ejercicios prácticos y colaborativos apoyados o no en la tecnología. Se persigue con ello que los estudiantes desarrollen sus competencias digitales a través de la creación de contenidos educativos con soporte en las herramientas tecnológicas y su planificación y desarrollo tecnopedagógico en un entorno de enseñanza secundaria. Para ello se utilizan diversas herramientas de creación de contenidos, evaluación de actividades, rúbricas, etc.

Todas las actividades se han diseñado pensando en el aumento de la competencia digital de los estudiantes (futuros docentes o docentes en servicio de educación secundaria o formación profesional). Se estableció una relación entre estos criterios de evaluación, es decir, entre las rúbricas de evaluación de las actividades, y las dimensiones establecidas en el portafolio de CDD elaborado por el INTEF (2017). Por lo tanto, se presupone que, con una adecuada realización de estas actividades, el estudiante podrá progresar en las distintas áreas de Competencia Digital Docente.

\section{Las competencias digitales docentes a desarrollar}

De acuerdo a la naturaleza de la asignatura de Complementos para la Formación Disciplinar de la especialidad Tecnología e Informática de la maestría en formación del profesorado, en las actividades se han propuesto tareas vinculadas a las áreas de competencia digital que se refieren en INTEF (2017):

Información: Todas las acciones relacionadas con la identificación, localización, organización y recuperación de información, así como su evaluación y relevancia según sus fines.

Comunicación y colaboración: Las actividades relacionadas con la interacción y la comunicación en los entornos web, mediante la conexión, colaboración y participación en comunidades de aprendizaje y las redes sociales digitales.

Creación de contenidos: Producir y editar contenidos educativos digitales en los diversos formatos posibles, así como rediseñar, modificar y transformar contenidos existentes en nuevas versiones, teniendo en cuenta los derechos de autoría y el licenciamiento Creative Commons. 
Resolución de problemas: Identificar y decidir qué recursos digitales deben ser utilizados según la necesidad planteada. Así también, resolver problemas a través de estos recursos y usar las herramientas de forma creativa. Solucionar también los problemas técnicos.

El área 4 (Seguridad) del portafolio de competencia digital docente que propone el INTEF se ha quedado fuera del diseño de las actividades porque no es objeto de la materia de Complementos para la formación disciplinar en el contexto de la maestría en Formación del Profesorado.

La competencia digital, al igual que cualquier adquisición de competencias, debe ser demostrada con la evidencia de su logro. De este modo, se considera que un estudiante alcanza una competencia cuando emite y entrega una evidencia de ello, tal y como se ha explicado anteriormente en la descripción de las actividades. Luego, estas evidencias se pueden constatar a través del uso de rúbricas de evaluación.

\section{Validación de las rúbricas de evaluación de las actividades formativas}

Como se mencionó anteriormente, se crearon rúbricas de valoración de las actividades en las que se relacionaban los criterios de evaluación con los indicadores de las competencias digitales docentes establecidas por el INTEF, 2017 (Figura 1). Para la validación de estas rúbricas se procedió en dos fases. En la primera de estas, se validaron las rúbricas con respecto a la dimensión de Competencia Digital Docente que se quiere trabajar, a través del procedimiento del juicio de expertos.

\section{Figura 1}

Criterios de evaluación de las rúbricas de las e-actividades relacionados con el modelo de la competencia digital docente (INTEF, 2017) 
Competencia digital docente (INTEF,2017)

\begin{tabular}{|c|c|c|}
\hline $\begin{array}{l}\text { Área de la competencia } \\
\text { digital }\end{array}$ & $\begin{array}{l}\text { Indicador de la } \\
\text { competencia }\end{array}$ & Criterio de evaluación en la rúbrica de la e-actividad \\
\hline \multirow{5}{*}{$\begin{array}{l}\text { Información y } \\
\text { alfabetización } \\
\text { informacional }\end{array}$} & \multirow{2}{*}{$\begin{array}{l}\text { Navegación, } \\
\text { búsqueda y filtrado } \\
\text { de información }\end{array}$} & $\begin{array}{l}\text { A1.La actividad releccionada se relaciona con el fomento de las vocaciones STEM. Se realiza un } \\
\text { análisis profundo con argumentos que lo sostiene. }\end{array}$ \\
\hline & & $\begin{array}{c}\text { A3.Se indican fuentes de información en su PLE y se muestra actividad de gestión (organización, } \\
\text { clasificación etiquetas, etc.) }\end{array}$ \\
\hline & \multirow{2}{*}{$\begin{array}{l}\text { Evaluación de la } \\
\text { información }\end{array}$} & $\begin{array}{l}\text { A1.La actividad seleccionada se relaciona con el fomento de las vocaciones STEM. Se realiza un } \\
\text { análigis profundo con argumentos que lo sostiene. }\end{array}$ \\
\hline & & $\begin{array}{l}\text { A3.Se indican fuentes de información en su PLE y se muestra actividad de gestión (organización, } \\
\text { clasificación etiquetas, etc.) }\end{array}$ \\
\hline & $\begin{array}{l}\text { Almacenamiento y } \\
\text { recuperación de } \\
\text { información }\end{array}$ & $\begin{array}{l}\text { A3.Se indican fuentes de información en su PLE y se muestra actividad de gestión (organización, } \\
\qquad \text { clasificación etiquetas, etc.) }\end{array}$ \\
\hline \multirow{7}{*}{$\begin{array}{l}\text { Comunicación y } \\
\text { colaboración }\end{array}$} & \multirow{4}{*}{$\begin{array}{l}\text { Interacción mediante } \\
\text { nuevas tecnologías }\end{array}$} & A1.Se Incluye el hashtag \#Msec_Tel_STEM \\
\hline & & A2.Se Incluye el hashtag \#Msec_Tel_Twitter \\
\hline & & A3. Se crea contenido y se difunde y comparte. \\
\hline & & $\begin{array}{l}\text { A4. Hay evidencias de trabajo grupal con comunicación online. Todos los componentes participan } \\
\text { de forma activa. Se muestra implicación en la tarea. }\end{array}$ \\
\hline & $\begin{array}{l}\text { Compartir } \\
\text { información y }\end{array}$ & $\begin{array}{l}\text { A1. Se comparte por Twitter el enlace del análisis realizado y publicado (en plataforma de libre } \\
\qquad \begin{array}{c}\text { acceso). }\end{array}\end{array}$ \\
\hline & contenidos & $\begin{array}{l}\text { A2.Se comparte por Twiitter el enlace de la actividad creada y publicada (en Scribd u otra } \\
\text { plataforma de libre acceso). }\end{array}$ \\
\hline & $\begin{array}{l}\text { Colaboración } \\
\text { mediante canales } \\
\text { digitales }\end{array}$ & $\begin{array}{l}\text { A4. Hay evidencias de trabajo grupal con comunicación online. Todos los componentes participan } \\
\qquad \text { de forma activa. Se muestra implicación en la tarea. }\end{array}$ \\
\hline \multirow{9}{*}{$\begin{array}{c}\text { Creación de contenido } \\
\text { digital }\end{array}$} & \multirow{5}{*}{$\begin{array}{l}\text { Desarrollo de } \\
\text { contenidos }\end{array}$} & A3.Se crea contenido y se difunde y comparte. \\
\hline & & A4.Incluye todas las partes de la webquest con sus especificaciones. \\
\hline & & $\begin{array}{l}\text { A5. Crea/edita vídeos y/o podcast o diverso material multimedia para antes de la clase con } \\
\text { Flipped Classroom. }\end{array}$ \\
\hline & & $\begin{array}{l}\text { A5.Establece rúbrica de evaluación y coevaluación y emplea aplicaciones digitales (Corubric, } \\
\text { rubristars u otra) para la clase con Flipped Classroom }\end{array}$ \\
\hline & & $\begin{array}{l}\text { A5.Presenta acciones para mejorar la retención y la transferencia (quiz online, reflexión, } \\
\text { metacognición, etc.). }\end{array}$ \\
\hline & \multirow{2}{*}{$\begin{array}{l}\text { integración y } \\
\text { reelaboración }\end{array}$} & $\begin{array}{l}\text { A3.Indicar algún tipo de esquema, mapa mental u organizador gráfico en el que se vea la } \\
\text { constelación de tu PLE. }\end{array}$ \\
\hline & & $\begin{array}{l}\text { A5. Crea/edita vídeos } y / 0 \text { podcast o diverso material multimedia para antes de la clase con } \\
\text { Flipped Classroom. }\end{array}$ \\
\hline & \multirow{2}{*}{$\begin{array}{l}\text { Derechos de autor y } \\
\text { licencias }\end{array}$} & A1.Indica datos de referencia completos \\
\hline & & A2.Indica datos de referencia completos \\
\hline \multirow{9}{*}{ Solución de problemas } & $\begin{array}{l}\text { Identificación de } \\
\text { necesidades y } \\
\text { respuestas tecnológicas }\end{array}$ & $\begin{array}{l}\text { A3.Incluir una breve exposición de las ventajas, dificultades, obatáculos, etc. que has encontrado } \\
\qquad \text { en el momento de diseñar tu PLE. }\end{array}$ \\
\hline & \multirow{7}{*}{$\begin{array}{l}\text { Innovar y utilizar la } \\
\text { tecnología de forma } \\
\text { creativa }\end{array}$} & $\begin{array}{l}\text { A2.La actividad planteada se relaciona directamente con la especialidad de Tecnología e } \\
\text { Informática. Incluye el uso de un hashtas. Se establece claramente el uso de Twitter en la } \\
\text { actividad planteada. }\end{array}$ \\
\hline & & $\begin{array}{l}\text { A3. Incluir una breve conclusión de cuáles son las utilidades y aplicaciones que tiene el uso del PLE } \\
\text { en las aulas de Secundaria, bachillerato, formación profesional o idiomas ( según sea tu caso) }\end{array}$ \\
\hline & & $\begin{array}{l}\text { A4. La webquest creada motiva a los alumnos a la investigación guiada, la elaboración de } \\
\text { productos o proyectos con base en herramientas digitales y fomenta el trabajo cooperativo. }\end{array}$ \\
\hline & & A5.Presenta instrucciones + estimulos para antes de la clase con Flipped Classroom. \\
\hline & & $\begin{array}{l}\text { A5.Presenta actividad de orientación + actividad de clase para estimular respuestas y } \\
\text { participación + Acción de retroalimentación o refuerzo durante la clase con Flipped Classroom }\end{array}$ \\
\hline & & $\begin{array}{l}\text { A5. Establece rúbrica de evaluación y coevaluación y emplea aplicaciones digitales (Corubric, } \\
\text { rubristars u otra) para la clase con Flipped Classroom }\end{array}$ \\
\hline & & $\begin{array}{l}\text { A5.Presenta acciones para mejorar la retención y la transferencia (quiz online, reflexión, } \\
\text { metacognición, etc.). }\end{array}$ \\
\hline & $\begin{array}{l}\text { Identificación de } \\
\text { lagunas en la } \\
\text { competencia digital }\end{array}$ & $\begin{array}{l}\text { A3.Incluir una breve exposición de las ventajas, dificultades, obstáculos, etc. que has encontrado } \\
\qquad \text { en el momento de diseñar tu PLE. }\end{array}$ \\
\hline
\end{tabular}

Para ello se elaboró una encuesta utilizando la plataforma Google Forms en la que se relacionaban los criterios de evaluación de las rúbricas de las actividades formativas con los 
indicadores de las competencias digitales docentes. Los jueces debían establecer si esa relación cumplía con tres criterios: claridad, idoneidad y relevancia y se pedía a los expertos escoger en una escala de 1-4 la pertinencia del criterio con la dimensión señalada (ver ejemplo juez 1: https://doi.org/10.5281/zenodo.3715482).

La encuesta fue enviada a 6 profesores universitarios del ámbito de educación. Todos ellos poseen experiencia en investigación y docencia en tecnología educativa. De los 6 jueces evaluadores, 2 eran hombres y 4 mujeres, con una experiencia docente superior a 3 años en docencia universitaria. En el mismo formulario se les invitaba a realizar una valoración cualitativa de los diversos ítems.

Una vez recibidos los formularios completos, se procedió a calcular el grado de concordancia entre los jueces a través del coeficiente Kappa de Cohen, indicado para establecer la validez de contenido (Altman, 1991; Escobar-Pérez y Cuervo-Martínez 2008). Cada criterio fue evaluado de forma individual y en cada indicador de las áreas de la competencia digital marcadas según su actividad formativa; se procedió a aceptar dicho criterio cuando había una "buena" o "muy buena" concordancia ( 0.60 a 0.8 o 0.8 a 1 respectivamente) entre los jueces (Altman,1991; López-de-Ullibarri y Pita-Fernández, 1999) cuando se emplea el índice de Kappa para establecer la validación de contenido (ver Tabla 2).

En la segunda fase, se procedió a validar estas rúbricas de forma empírica. Para cada actividad propuesta, se seleccionaron varias entregas de los estudiantes (entre 8 y 10) que fueron corregidas por tres profesores; estos se basaron en las rúbricas para asignar la puntuación a las actividades de los estudiantes. Con las puntuaciones obtenidas se calculó la variabilidad entre ellos a través de la media y desviación estándar (ver Tabla 3).

\section{Resultados}

En la tabla 1 se han asociado las cinco actividades planteadas con los indicadores de las diferentes dimensiones de la Competencia digital docente (INTEF, 2017). 


\section{Tabla 1}

Correspondencia entre los distintos indicadores de cada una de las dimensiones marcadas en el marco común de Competencia Digital Docente (INTEF, 2017) y las actividades diseñadas

\begin{tabular}{|c|c|c|}
\hline & $\begin{array}{l}\text { Indicadores } \\
\text { Competencias }\end{array}$ & Actividades \\
\hline \multirow{5}{*}{$\begin{array}{l}\text { Dimensión 1: Información y } \\
\text { Alfabetización informacional }\end{array}$} & 1.1 & A1 \\
\hline & 1.1 & A3 \\
\hline & 1.2 & A1 \\
\hline & 1.2 & A3 \\
\hline & 1.3 & A3 \\
\hline \multirow{7}{*}{$\begin{array}{c}\text { Dimensión 2: Comunicación y } \\
\text { colaboración }\end{array}$} & 2.1 & A1 \\
\hline & 2.1 & $\mathrm{~A} 2$ \\
\hline & 2.1 & A3 \\
\hline & 2.1 & A4 \\
\hline & 2.2 & A1 \\
\hline & 2.2 & $\mathrm{~A} 2$ \\
\hline & 2.4 & A4 \\
\hline \multirow{9}{*}{$\begin{array}{l}\text { Dimensión 3: Creación de } \\
\text { contenidos digitales }\end{array}$} & 3.1 & A3 \\
\hline & 3.1 & A4 \\
\hline & 3.1 & A5 \\
\hline & 3.1 & A5 \\
\hline & 3.1 & A5 \\
\hline & 3.2 & A3 \\
\hline & 3.2 & A5 \\
\hline & 3.3 & A1 \\
\hline & 3.3 & $\mathrm{~A} 2$ \\
\hline \multirow{4}{*}{$\begin{array}{c}\text { Dimensión 5: Resolución de } \\
\text { problemas }\end{array}$} & 5.1 & A3 \\
\hline & 5.2 & $\mathrm{~A} 2$ \\
\hline & 5.2 & A3 \\
\hline & 5.2 & A4 \\
\hline
\end{tabular}




$\begin{array}{ll}5.2 & \text { A5 } \\ 5.2 & \text { A5 } \\ 5.2 & \text { A5 } \\ 5.2 & \text { A5 } \\ 5.3 & \text { A3 }\end{array}$

Los resultados de la validación de la relación entre las actividades y los indicadores de la competencia digital docente se muestran en la tabla 2. Para ello se calculó el índice de Kappa de concordancia entre los jueces.

\section{Tabla 2}

Valores calculados para el indicador de concordancia entre jueces de Kappa para cada dimensión examinada

\begin{tabular}{lccc}
\hline & Claridad & Idoneidad & Relevancia \\
\hline Dimensión 1 & 0,80 & 0,76 & 0,69 \\
Dimensión 2 & 0,86 & 0,76 & 0,76 \\
Dimensión 3 & 0,90 & 0,90 & 0,83 \\
Dimensión 5 & 0,78 & 0,81 & 0,76 \\
\hline
\end{tabular}

Como se observa en la Tabla 2, la concordancia entre jueces es muy buena en el criterio de Claridad ( $\mathrm{K}>0,80$ en la mayoría de los casos); buena o muy buena en el criterio de idoneidad $(0,75>K>0,9)$ y buena para el criterio de relevancia $(0,60<\mathrm{k}<0,80)$. Con respecto a la evaluación cualitativa no hubo comentarios sobre modificación de algún indicador, por lo que se aceptaron los indicadores tal y como aparecían en la rúbrica.

Tras esta primera validación, se procedió a realizar la validación empírica de las rúbricas. Para ello, se seleccionaron entre 8 y 10 trabajos de estudiantes para cada actividad que fueron corregidos por tres profesores diferentes. Se consideró el promedio de calificaciones en cada actividad según cada juez. En la tabla 3 se muestran los resultados obtenidos.

Tabla 3

Puntuaciones otorgadas para cada una de las actividades y estadísticos relacionados 


\begin{tabular}{llllll}
\hline & A1 & A2 & A3 & A4 & A5 \\
\hline J1 & 7.1 & 7.8 & 7.0 & 9.1 & 8.8 \\
J2 & 9.5 & 9.1 & 10.0 & 10.0 & 8.6 \\
J3 & 8.2 & 9.6 & 7.0 & 7.8 & 8.5 \\
Media & 8.3 & 8.2 & 8.1 & 9.0 & 8.6 \\
Desviación & 1.4 & 1.5 & 1.7 & 1.4 & 0.9 \\
típica & & & & & \\
\hline
\end{tabular}

Como se puede observar en la Tabla 3, la variación entre las puntuaciones otorgadas para cada trabajo por los evaluadores tiene una desviación típica entre las distintas evaluaciones de entre 0,9 y 1,7 para las distintas actividades. El Juez 2 otorgó una calificación más alta con respecto a los otros dos, debido a que este calificador fue el mismo profesor que impartía la asignatura, por lo que pudo tener en cuenta otros factores además del trabajo entregado. Posteriormente, se realizó una comparación entre las puntuaciones otorgadas en la evaluación de las actividades por los distintos jueces aplicando la prueba $\mathrm{F}$ de Friedman con una significación de 0,05. En la tabla 4 se muestran los resultados respectivos.

\section{Tabla 4}

Significación encontrada al realizar la comparación de medias con la prueba $\mathrm{F}$ de Friedman.

\begin{tabular}{llcc}
\hline & J1-J2 & J1-J3 & J2-J3 \\
\hline Actividad 1 & 0,020 & 0,157 & 0,096 \\
\hline Actividad 2 & 0,014 & 0,705 & 0,414 \\
\hline Actividad 3 & 0,005 & 0,739 & 0,005 \\
\hline Actividad 4 & 0,046 & 0,034 & 0,008 \\
\hline Actividad 5 & 0,137 & 1,000 & 0,739 \\
\hline
\end{tabular}

Como se puede observar en la tabla 4 , la calificación otorgada por el Juez 1 y 3 no difiere estadísticamente (medias iguales) para las actividades 1, 2, 3, y 5. También se encontró significación entre el Juez 2 y el Juez 3 en la actividad 2 y todos los Jueces estuvieron de acuerdo 
en la calificación de la actividad 5. Las calificaciones otorgadas en la actividad 4 son diferentes para los tres jueces.

\section{Discusión}

Son numerosos los estudios que tratan de analizar la evolución de la competencia digital docente en la formación y desempeño de maestros y profesores, debido a la gran implicación que esta tiene para su futuro desempeño laboral (Pérez-Escoda y Rodríguez-Conde, 2016; Ferrando, Hurtado y Beltrán, 2018; Sánchez-Otero et al. 2019). En muchos casos apuntan a la necesidad de establecer actividades en el currículum que vayan encaminadas al desarrollo de dicha competencia. La asignatura de Complementos de Formación de la especialidad de Tecnología e Informática ha sido diseñada para facilitar este desarrollo gracias a las eactividades preparadas para la evaluación continua.

Con las e-actividades descritas y las rúbricas de evaluación que los estudiantes tenían a su disposición desde el inicio, se contribuyó al desarrollo de las respectivas dimensiones: 1, Información y Alfabetización informacional; 2, Comunicación y Colaboración; 3, Creación de contenidos digitales; y 4, Resolución de problemas.

Por otro lado, el proceso de validación de las rúbricas a través del juicio de expertos resultó favorable en todos los casos (Kappa igual o mayor a 0.75), considerando los criterios de claridad, idoneidad y relevancia en relación con la competencia digital que se quería trabajar. Según los índices de concordancia entre los jueces se puede afirmar que las rúbricas de evaluación de las actividades tienen la validez suficiente para considerar que corresponden con la evaluación de las competencias digitales abordadas en las actividades formativas; es decir, se puede afirmar que están conformes con la premisa de que la evaluación de estas actividades también se corresponde con la evaluación de la competencia digital de estos estudiantes.

Finalmente, en los resultados de validación empírica en la cual se contrastaron los resultados de la evaluación de las e-actividades por parte de tres profesores-basándose estos en las rúbricas de evaluación-, se encontró que la mayor dispersión obtenida entre las evaluaciones de los tres profesores es de 1,7 en la desviación típica (>20\%). Hay que destacar que uno de los profesores era el que impartía la asignatura. En las notas obtenidas por este docente, se observa un sesgo positivo; esto queda demostrado al aplicar la prueba F de Friedman para analizar la diferencia de medias. Como se ha indicado, los jueces 1 y 3 demostraron no haber diferencia de medias en las actividades 1, 2, 3 y 5 . No obstante, el caso de la actividad 4 merece una 
revisión posterior, tanto la descripción de la actividad como la rúbrica asociada para mejorar esa desviación observada en las correcciones; aunque también hay que considerar que era una actividad de mayor nivel de complejidad y además colaborativa. El juez 2 evaluó, como se observa en la tabla 2, al alza las distintas actividades, teniendo en cuenta otros factores. Esto quiere decir que las rúbricas utilizadas sirven para evaluar la evolución de la competencia digital docente.

\section{Referencias}

Alsina, J., Irurita, A., Trenchs, M. A., Miró, M. B., Marín, A. C., Busquets, M. C., y Ruiz, L. M. (2013). Rúbricas para la evaluación de competencias. Octaedro. Cuadernos de docencia universitaria, $26 . \quad$ Recuperado de: http://www.ub.edu/ice/sites/default/files/docs/qdu/26cuaderno.pdf

Altman D. (1991). Practical statistics for medical research. New York: Chapman and Hall.

Álvarez, J. F. y Gisbert, M. (2015). Information Literacy Grade of Secondary School Teachers in Spain - Beliefs and Self-Perceptions. Comunicar, 45, 187-194. https://doi.org/10.3916/C45-2015-20

Area, M. (2015). Reinventar la escuela en la sociedad digital. Del aprender repitiendo al aprender creando. Mejorar los aprendizajes en la educación obligatoria. Políticas y actores. Buenos Aires: Instituto Internacional de Planeamiento de la Educación IIPEUnesco. Recuperado de: http://unesdoc.unesco.org/images/0023/002349/234977s.pdf

Area, M., y González, C. (2015). De la enseñanza con libros de texto al aprendizaje en espacios online gamificados. Educatio Siglo XXI, 33(3), 15. https://doi.org/10.6018/j/240791

Blau, I., Grinberg, R., y Shamir-Inbal, T. (2018). Pedagogical perspectives and practices reflected in metaphors of learning and digital learning of ICT leaders. Computers in the Schools, 35(1), 32-48. https//doi.org/10.1080/07380569.2018.1427960

Brown, S. (2015). La evaluación auténtica: el uso de la evaluación para ayudar a los estudiantes a aprender. RELIEVE - Revista electrónica de investigación y evaluación educativa, 21(2), 1-11. https://doi.org/10.7203/relieve.21.2.7674

Durán, M., Gutiérrez, I., y Paz, M. (2016) Análisis conceptual de modelos de competencia digital del profesorado universitario. Relatec: Revista latinoamericana de tecnología educativa, 15(1), 97-114. DOI: https://doi.org/10.17398/1695-288X.15.1.97

Escobar-Pérez, J., y Cuervo-Martínez, Á. (2008). Validez de contenido y juicio de expertos: una aproximación a su utilización. Avances en medición, 6(1), 27-36. Recuperado de: https://www.researchgate.net/publication/302438451_Validez_de_contenido_y_juicio de_expertos_Una_aproximacion_a_su_utilizacion

Ferrando, I., Hurtado, D. y Beltrán, M. (2018). Formación STEM en el grado de maestro: una experiencia docente. Revista d' Innovació educativa, 20, 35-43. Recuperado de: https://ojs.uv.es/index.php/attic/article/view/10946/11794 
García-Ros, R. (2011). Análisis y validación de una rúbrica para evaluar habilidades de presentación oral en contextos universitarios. Electronic journal of research in $\begin{array}{lll}\text { educational psychology, } & 9(3), & 1043-1062\end{array}$ https://www.redalyc.org/pdf/2931/293122852004.pdf

INTEF. (2017) Marco común de competencia digital docente del plan de cultura digital en la escuela. Madrid, España: Ministerio de Educación, Cultura y Deporte. Recuperado de: https://www.slideshare.net/educacionlab/marco-comn-de-competencia-digitaldocente-2017

López-de Ullibarri, I., y Pita-Fernández, S. (1999). Medidas de concordancia: El índice Kappa. Cuadernos de atención primaria, 6(4), 223-226. Recuperado de: https://www.fisterra.com/mbe/investiga/kappa/kappa2.pdf

Martín, D., Sáenz, M., Santiago, R., y Chocarro, E. (2016). Diseño de un instrumento para evaluación diagnóstica de la competencia digital docente: formación flipped classroom. Revista DIM: Didáctica, innovación y multimedia, 33, Recuperado de: https://ddd.uab.cat/record/148407

Pérez-Escoda, A, y Rodríguez-Conde, M. J. (2016). Evaluación de las competencias digitales autopercibidas del profesorado de educación primaria en Castilla y León (España). Revista de investigación educativa, 34(2), 399-415. DOI: https://doi.org/10.6018/rie.34.2.215121

Pérez, M. S., y Flores, M. (2017). Los criterios de evaluación, como un apoyo para los estudiantes en el logro de las competencias en cursos en línea. Campus virtuales, 6(2), 9-20. Recuperado de: http://www.uajournals.com/campusvirtuales/journal/11/1.pdf

Salazar-Goméz, E., Tobón, S. y Juárez-Hernández, L. (2018). Diseño y validación de una rúbrica de evaluación de las competencias digitales desde la socioformación. Apuntes Universitarios, 8(3). https://doi.org/10.17162/au.v8i3.329

Sánchez-Otero, M., García-Guiliany, J., Steffens-Sanabria, E., y Hernández-Palma, H (2019). Estrategias pedagógicas en procesos de enseñanza y aprendizaje en la educación superior incluyendo tecnologías de la información y las comunicaciones. Información tecnológica, 30(3), 277-286. https $/ / d x . d o i . o r g / 10.4067 / S 0718-07642019000300277$

Silva, J. (2017). Un modelo pedagógico virtual centrado en las e-actividades. Revista de educación a distancia, 53. DOI: http://dx.doi.org/10.6018/red/53/10

Silva, W. H. y Mazuera, J. A. (2019). ¿Enfoque de competencias o enfoque de capacidades en la escuela? Revista electrónica de investigación educativa, 21, e17, 1-10. doi.10.24320/die.2019.21.e17.1981

Tourón, J., Martín, D., Asencio, E., Pradas, S., e Íñigo, V. (2018). Validación de constructo de un instrumento para medir la competencia digital docente de los profesores (CDD). Revista española de pedagogía, 25-54.

Tourón, J., y Martín, D. (2019). Aprender y enseñar en la universidad hoy. Guía práctica para profesores. UNIR editorial 